\title{
Clinical Characteristics in Patients with Hospital- Acquired Pneumonia Caused by Extensively Drug- Resistant Acinetobacter baumannii, A Multicenter Retrospective Study
}

\section{Yujun Li}

South China University of Technology School of Medicine

Xiaomei Huang

South China University of Technology School of Medicine

Yuyao Wang

Danbury Hospital

\section{Chuzhi Pan}

al Third Affiliated Hospitof Sun Yat-Sen University

\section{Zexun Mo}

South China University of Technology School of Medicine

\section{Muchao Huang}

Guangzhou Xinhai Hospital

\section{Changquan Fang}

Guangzhou Red Cross Hospital

\section{Penghao Guo}

Sun Yat-sen University First Affiliated Hospital

\section{Huiling Chen}

South China University of Technology School of Medicine

\section{Zhuxiang Zhao}

South China University of Technology School of Medicine

\section{Ziwen Zhao}

South China University of Technology School of Medicine

Shuquan Wei ( $\square$ eyweishuquan@scut.edu.cn )

Guangzhou First People's Hospital https://orcid.org/0000-0003-4044-2244

\section{Research article}

Keywords: Acinetobacter baumannii, Extensive drug resistance, Pneumonia, multicenter retrospective study 
Posted Date: December 1st, 2020

DOI: https://doi.org/10.21203/rs.3.rs-116166/v1

License: (c) (i) This work is licensed under a Creative Commons Attribution 4.0 International License. Read Full License 


\section{Abstract}

Background Extremely drug-resistant (XDR) Acinetobacter baumannii $₫ A$. baumanni» concern. The relationship between XDR and patient outcomes remains unclear. We investigated the clinical features, risk factors, and outcomes of Hospital-acquired pneumonia (HAP)caused by XDR $A$. baumannii.

Methods A multicenter retrospective case-control study was performed to determine factors associated with XDR A. baumannii pneumonia from 5 teaching hospitals in Guangzhou, China.

Results 76 patients were enrolled in the study. XDR A. baumannii pneumonia patients were tend to be smoker $(11.9 \%$ vs $3.9 \%, P=0.130)$ and older $(76.5 \pm 11.2$ vs $70.3 \pm 16.4, P=0.007)$ and had more comorbid diseases including chronic obstructive pulmonary disease (COPD) (48.7\% vs $21.1 \%, P=0.001)$ and renal failure $(21.1 \%$ vs $3.9 \%, P=0.002)$ and had higher APACHE II score $(65.8 \%$ vs $47.4 \%, P=0.033)$. Invasive procedures including insertion of urinary catheter, nasogastric tube, central venous/arterial catheter, bronchoscopy and mechanical ventilation along with using $\beta$-lactam/ $\beta$-lactamase inhibitor and carbapenem were also risk factors for XDR A. baumannii pneumonia. Multivariate analysis showed the APACHE II score $>=20$ (OR, 2.1; 95\% Cl: 1.1-4.1, P = 0.023), COPD (OR, 9.6; 95\% Cl: 2.0-45.5, P = 0.004), central venous/arterial catheter placement $(\mathrm{OR}, 11.5 ; 95 \% \mathrm{Cl}: 1.1-117.8, \mathrm{P}=0.040)$, low albumin levels (OR, $1.2 ; 95 \% \mathrm{Cl}: 1.1-1.4, \mathrm{P}=0.001)$ and using $\beta$-lactam/ $\beta$-lactamase inhibitor $(\mathrm{OR}, 15.9 ; 95 \% \mathrm{Cl}: 2.7-94.2, \mathrm{P}=$ 0.002) were independent risk factors for XDR A. baumannii pneumonia. Compared with the non-XDR $A$. baumannii patients, the XDR $A$. baumannii pneumonia increased length of mechanical ventilation (11.1 \pm 12.3 vs $5.1 \pm 5.6, P=0.000)$, hospital stay $(42.2 \pm 24.3$ vs $34.8 \pm 18.0, P=0.036)$ and ICU (Intensive Care Unit) stay $(27.5 \pm 19.0$ vs $20.0 \pm 20.5, P=0.020)$, but it did not increase in-hospital mortality $(47.4 \%$ vs $32.9 \%, P=0.137)$.

Conclusions XDR A. baumannii pneumonia was strongly related to systemic illnesses, invasive procedure, low albumin levels and the APACHE II score and increasing the length of mechanical ventilation and hospital stay. But it did not increase in-hospital mortality.

\section{Background}

Acinetobacter baumannii (A. baumannii) is one of the most troublesome pathogens causing Hospitalacquired pneumonia (HAP) worldwide[1-3]. Due to its remarkable ability to acquire antibiotic resistance, A. baumannii infection is also difficult to control and treat[4-6]. CHINET surveillance system from China showed $A$. baumannii resists to many powerful antimicrobial agents, especially imipenem and meropenem which were once considered as the "gold standard"[7]. And the resistance rate to those agents went up to $77.1 \%$ and $78.1 \%$ respectively in 2018[6]. Extremely drug-resistant (XDR) A. baumannii was defined as resistance to all available antibiotics except for colistin and tigecycline[8-10]. It became a major resistance phenotype, ranged from $68.3-72.9 \%$ of the reported $A$. baumannii strains[11, 12]. Colistin and tigecycline are believed to be the last few available choices for XDR infections. But 
tigecycline and polymyxin resistance $A$. baumannii had already been reported[5, 13-15]. XDR A. baumannii with limited treatment options was believed to be associated with high mortality and treatment failure and served as a marker of poor prognosis.

However, the relationship between antibiotic resistance of $A$. baumannii strains and outcomes caused with concern and dispute. One study in 2007 found mortality rates of patients with multi-drug resistant (MDR) A. baumannii were not higher than the patients without MDR A. baumannii [16]. Our previous study in 2017 was limited by the sample size, but it showed XDR A. baumannii pneumonia was not associated with in-hospital mortality[10]. Two studies in 2019 showed increasing mortality rate may relate to patient comorbidity and inappropriate therapy, but not antibiotic resistance $[12,17]$. It is frustrated for clinicians to treat XDR A. baumannii and the data for XDR A. baumannii HAP was still limited. We believe the relationship between XDR A. baumannii pneumonia and outcomes needs further investigation. Here, we conducted a multicenter study to investigate the clinical features, risk factors, and outcomes of HAP caused by XDR A. baumannii from 5 teaching hospitals in Guangzhou, China.

\section{Methods}

\section{Participants}

A multicenter retrospective case-control study was conducted to evaluate the clinical features, risk factors, and outcomes of HAP caused by XDR A. baumannii from 5 teaching hospitals in Guangzhou, China. GFPH, Guangzhou First People's Hospital, the Second Affiliated Hospital of South China University of Technology, Pan Fu Road, Guangzhou, China; TAH, the Third Affiliated Hospital of Sun Yat-sen University, Tian He Road, Guangzhou, China; FAH, the First Affiliated Hospital of Sun Yat-sen University, Zhong Shan Er Road, Guangzhou, China; GXH, Guangzhou Xinhai Hospital, Xin Gang west Road, Guangzhou, China; GRCH, Guangzhou Red Cross Hospital, Tong Fu zhong Road, Guangzhou, China. The participants included patients who developed HAP and ventilator-associated pneumonia (VAP) [12] from XDR A. baumannii (confirmed with respiratory samples) from April 2011 to April 2016. The controls, matched to the cases by hospitals, were patients diagnosed with non-XDR A. baumannii HAP and randomly selected within the 5 teaching hospitals during the studying period.

\section{Inclusion and exclusion criteria}

The inclusion criteria consisted of the following:

1, Diagnostic criteria for pneumonia[18]: new or progressive pulmonary infiltrates in chest X-ray or CT imaging, plus at least two of the following supportive clinical signs: temperature of $>38^{\circ} \mathrm{C}$ or $<35.5^{\circ} \mathrm{C}$, leukocytosis of $>12,000$ or $<4000 \mathrm{WBC} / \mathrm{mm}^{3}$, purulent respiratory secretions, or worsening oxygenation determined by arterial oxygen tension/fraction of inspired oxygen ratio $\left(\mathrm{PaO}_{2} / \mathrm{FiO}_{2}\right)$. 
2, At least two positive clinical respiratory cultures for A. baumannii: protected brushing specimen $10^{3}$ $\mathrm{CFU} / \mathrm{ml}$, bronchoalveolar lavage (BAL) fluid specimen $10^{4}$ or $10^{5} \mathrm{CFU} / \mathrm{ml}$, or endotracheal aspirate specimen $10^{6} \mathrm{CFU} / \mathrm{ml}$.

3, Antimicrobial susceptibility testing shows resistance to all available antibiotics except for colistin and tigecycline.

The exclusion criteria consisted of the following:

1, Patient was less than 18 years of age.

2 , The length of hospitalization was less than 48 hours.

3, Patient had incomplete medical records.

4, Patient had other non- $A$. baumannii - positive respiratory cultures.

\section{Data acquisition}

A specially designed case record form (CRF) was used to collect the patient's clinical parameters, including age, gender, cigarette smoking, substance use history, laboratory test results (white blood cell count, platelet count, hemoglobin, creatinine, CRP, ESR and albumin), past medical diseases, invasive procedures (venous or arterial catheter placement, bronchoscopy, mechanical ventilation, et al.), the length of hospital stay (including ICU), pharmaceutical records (including antibiotic exposure), microbiological records (bacterial etiology, culture, and antimicrobial susceptibility testing results) and inhospital mortality. The Acute Physiology and Chronic Health Evaluation (APACHE) II score was used to determine the severity of diseases on patient's admission. The in-hospital mortality was compared between the HAP with and without XDR A. baumannii infection groups. The Guangzhou First People's Hospital, the Second Affiliated Hospital of South China University of Technology Ethics Committee approved the study.

\section{Statistical analysis}

SPSS (version 18.0) was used for statistical analysis. A P value less than 0.05 was considered statistically significant. Categorical variables were compared using the Chi-square test with Yates correction or Fisher's exact test. Continuous variables were analyzed using the two-tailed t-test. A multivariate logistic regression model was used to test the independent risk factors for XDR A. baumannii pneumonia.

\section{Results}


Based on the inclusion and exclusion criteria, a total of 76 patients with XDR A. baumannii pneumonia were included in the case-control study. 53 were male and 23 were female. The mean age was 76.5-yearold with a standard deviation of 11.2 years (Table 1). To analyze the potential risk factors, 76 cases of patients were compared with 76 control patients. 
Table 1

Clinical characteristics in HAP patients with XDR A. baumannii and non-XDR A. baumannii

\begin{tabular}{|c|c|c|c|}
\hline & $\begin{array}{l}\mathrm{XDR} \\
\text { A. baumannii } \\
(\mathrm{N}=76)\end{array}$ & $\begin{array}{l}\text { Non-XDR } \\
\text { A. baumannii } \\
(\mathrm{N}=76)\end{array}$ & P-value \\
\hline Age, $y^{*}$ & $76.5 \pm 11.2$ & $70.3 \pm 16.4$ & 0.007 \\
\hline Gender (M/F), $\mathrm{n}$ & $53 / 23$ & $48 / 28$ & 0.492 \\
\hline Cigarette smoking, n (\%) & $41(53.9)$ & $11(14.5)$ & 0.000 \\
\hline Alcohol abuse, $\mathrm{n}(\%)$ & $9(11.8)$ & $3(3.9)$ & 0.130 \\
\hline APACHE II score $>=20$ & $50(65.8)$ & $36(47.4)$ & 0.033 \\
\hline White blood cell count $\left(10^{9} / \mathrm{L}\right)^{\star}$ & $15.8 \pm 6.5$ & $13.0 \pm 5.0$ & 0.003 \\
\hline Platelet count $\left(10^{9} / \mathrm{L}\right)$ * & $161.4 \pm 77.5$ & $170.7 \pm 86.0$ & 0.486 \\
\hline Hemoglobin $(g / L)$ * & $102.5 \pm 22.0$ & $109.1 \pm 23.4$ & 0.074 \\
\hline Creatinine $(\mu \mathrm{mol} / \mathrm{L})$ * & $161.9 \pm 154.3$ & $126.4 \pm 109.5$ & 0.104 \\
\hline $\mathrm{CRP}(\mathrm{g} / \mathrm{L})$ * & $62.6 \pm 50.3$ & $69.2 \pm 58.0$ & 0.180 \\
\hline $\operatorname{ESR}(\mathrm{MM} / \mathrm{H})$ * & $50.1 \pm 39.8$ & $69.2 \pm 58.0$ & 0.486 \\
\hline Albumin $(g / L)$ * & $29.1 \pm 4.4$ & $41.8 \pm 31.5$ & 0.002 \\
\hline \multicolumn{4}{|l|}{ Time related to hospitalization* } \\
\hline Days of mechanical ventilation (days) & $11.1 \pm 12.3$ & $5.1 \pm 5.6$ & 0.000 \\
\hline Length of hospital stay before HAP (days) & $8.6 \pm 7.5$ & $8.8 \pm 8.1$ & 0.861 \\
\hline Length of stay in the ICU (days) & $27.5 \pm 19.0$ & $20.0 \pm 20.5$ & 0.020 \\
\hline Length of stay in the hospital (days) & $42.2 \pm 24.3$ & $34.8 \pm 18.0$ & 0.036 \\
\hline \multicolumn{4}{|l|}{ Associated disease, $\mathrm{n}(\%)$} \\
\hline COPD & $37(48.7)$ & $16(21.1)$ & 0.001 \\
\hline Diabetes mellitus & $26(22.4)$ & $33(43.4)$ & 0.318 \\
\hline Malignancy & $9(11.8)$ & $17(22.4)$ & 0.131 \\
\hline Coronary heart disease & $28(36.8)$ & $21(27.6)$ & 0.298 \\
\hline
\end{tabular}

*Values are presented as the mean \pm standard deviation; COPD, Chronic obstructive pulmonary disease; Malignancy includes haematological malignancies and solid tumours; CRP, C-reactive protein; ESR, Erythrocyte sedimentation rate; PPIs, proton pump inhibitor drugs. 


\begin{tabular}{|c|c|c|c|}
\hline & $\begin{array}{l}\mathrm{XDR} \\
\text { A. baumannii } \\
(\mathrm{N}=76)\end{array}$ & $\begin{array}{l}\text { Non-XDR } \\
\text { A. baumannii } \\
(\mathrm{N}=76)\end{array}$ & P-value \\
\hline Renal failure & $16(21.1)$ & $3(3.9)$ & 0.002 \\
\hline Cerebral haemorrhage & $8(10.5)$ & $7(9.2)$ & 1.000 \\
\hline \multicolumn{4}{|l|}{ Device, n (\%) } \\
\hline Urinary catheter & $76(100.0)$ & $65(85.5)$ & 0.001 \\
\hline Nasogastric tube & $76(100.0)$ & $62(81.6)$ & 0.000 \\
\hline Central venous/arterial catheter & $73(96.1)$ & $49(64.5)$ & 0.000 \\
\hline Bronchoscope & $65(85.5)$ & $51(67.1)$ & 0.012 \\
\hline Mechanical ventilation & $70(92.1)$ & $55(72.4)$ & 0.002 \\
\hline Tracheotomy & $7(9.2)$ & $4(5.3)$ & 0.533 \\
\hline \multicolumn{4}{|l|}{ Drug usage, n (\%) } \\
\hline Glucocorticoids & $47(61.8)$ & $35(46.1)$ & 0.073 \\
\hline PPIs & $61(82.9)$ & $56(82.9)$ & 0.441 \\
\hline \multicolumn{4}{|l|}{ Antimicrobial n (\%) } \\
\hline Cephalosporin & $37(48.7)$ & $42(55.3)$ & 0.516 \\
\hline Second generation & $11(14.5)$ & $14(18.4)$ & 0.662 \\
\hline Third generation & $30(39.5)$ & $33(43.4)$ & 0.742 \\
\hline$\beta$-lactam $/ \beta$-lactamase inhibitor & $71(93.4)$ & $52(68.4)$ & 0.000 \\
\hline Quinolone & $44(57.9)$ & $33(43.4)$ & 0.104 \\
\hline Aminoglycoside & $5(6.6)$ & $6(7.9)$ & 1.000 \\
\hline Macrolides & $2(2.6)$ & $0(0.0)$ & 0.497 \\
\hline Carbapenem & $35(46.1)$ & 14(18.4) & 0.000 \\
\hline Antimicrobial Combination therapy, $\mathrm{n}(\%)$ & $39(51.3)$ & $30(39.5)$ & 0.192 \\
\hline Mortality, n (\%) & $36(47.4)$ & 26(32.9) & 0.137 \\
\hline
\end{tabular}


Table 2

Multi-variate analysis of risk factors for XDR A. baumannii HAP patients

\begin{tabular}{|lll|}
\hline Risk factor & OR $(95 \%$ Cl $)$ & P-value \\
\hline APACHE II score $>=20$ & $2.1(1.1-4.1)$ & 0.023 \\
\hline COPD & $9.6(2.0-45.5)$ & 0.004 \\
\hline Central venous/arterial catheter & $11.5(1.1-117.8)$ & 0.040 \\
\hline Albumin & $1.2(1.1-1.4)$ & 0.001 \\
\hline B-lactam/ -lactamase inhibitor & $15.9(2.7-94.2)$ & 0.002 \\
\hline
\end{tabular}

The two groups were similar with respect to gender, substance use history, platelet count, hemoglobin, creatinine, CRP and ESR level. Additionally, no significant differences in the length of hospital stay before developing XDR A. baumannii pneumonia, history of diabetes mellitus, malignancy, coronary heart disease, renal failure, cerebral haemorrhage, tracheotomy, or the use of glucocorticoids, PPIs, cephalosporin, quinolone, macrolides, minoglycoside or antimicrobial combination therapy. While compared with the non-XDR A. baumannii patients, the patients with XDR A. baumannii pneumonia were more likely in smoking ( $11.9 \%$ vs $3.9 \%, P=0.130)$, older patients ( $76.5 \pm 11.2$ vs $70.3 \pm 16.4, P=0.007$ ), APACHE II score up to 20 and higher $(65.8 \%$ vs $47.4 \%, P=0.033)$, longer length of mechanical ventilation (11.1 \pm 12.3 vs $5.1 \pm 5.6, P=0.000)$, longer length of in hospital stay $(42.2 \pm 24.3$ vs $34.8 \pm 18.0, P=0.036)$ and ICU stay $(27.5 \pm 19.0$ vs $20.0 \pm 20.5, P=0.020)$, receiving more invasive procedure including insertion of urinary catheter $(100.0 \%$ vs $85.5 \%, P=0.001)$ and nasogastric tube $(100.0 \%$ vs $81.6 \%, P=0.000)$, central venous/arterial catheter placement $(96.1 \%$ vs $64.5 \%, \mathrm{P}=0.000)$, bronchoscopy $(85.5 \%$ vs $67.1 \%, \mathrm{P}$ $=0.012$ ) and mechanical ventilation ( $92.1 \%$ vs $72.4 \%, P=0.002)$. Moreover, patients with XDR $A$. baumannii pneumonia were more likely to have more comorbid diseases, such as chronic obstructive pulmonary disease (COPD) (48.7\% vs $21.1 \%, P=0.001)$ and renal failure $(21.1 \%$ vs $3.9 \%, P=0.002)$. Using $\beta$-lactam/ $\beta$-lactamase inhibitor ( $93.4 \%$ vs $68.4 \%, \mathrm{P}=0.001$ ) or carbapenem $(46.1 \%$ vs $18.4 \%, \mathrm{P}=$ 0.000 ) was also a risk factor for XDR A. baumannii pneumonia. But compared with the non-XDR $A$. baumannii patients, the XDR A. baumannii pneumonia did not increase in-hospital mortality $(47.4 \%$ vs $32.9 \%, \mathrm{P}=0.137)($ Tables 1$)$.

Multivariate analysis using a logistic regression model showed that the APACHE II score $>=20$ (OR, 2.1; 95\% Cl: 1.1-4.1, $\mathrm{P}=0.023), \mathrm{COPD}(\mathrm{OR}, 9.6 ; 95 \% \mathrm{Cl}: 2.0-45.5, \mathrm{P}=0.004)$, central venous/arterial catheter $(\mathrm{OR}, 11.5 ; 95 \% \mathrm{Cl}: 1.1-117.8, \mathrm{P}=0.040)$, lower albumin levels $(\mathrm{OR}, 1.2 ; 95 \% \mathrm{Cl}: 1.1-1.4, \mathrm{P}=0.001)$ and using $\beta$-lactam/ $\beta$-lactamase inhibitor $(\mathrm{OR}, 15.9 ; 95 \% \mathrm{Cl}$ : 2.7-94.2, $\mathrm{P}=0.002)$ were identified as independent risk factors for XDR $A$. baumannii pneumonia.

\section{Discussion}


XDR A. baumannii infection rate kept rising. But the antibiotics treatment options were very limited. It made the XDR A. baumannii one of the most troublesome pathogens worldwide. It seems preventing spreading the pathogen is the only reasonable way to reduce mortality. However, recently, the relationship between antibiotic resistance and outcomes caused with concern and dispute. Here, we presented this multicenter retrospective case-control study to investigate the clinical features, risk factors, and outcomes of HAP caused by XDR A. baumannii. It showed XDR A. baumannii pneumonia significantly increased the length of mechanical ventilation and hospital stay. But it was not associated with in-hospital mortality. Our finding indicated, besides antibiotics, we could improve outcomes if we optimize treatment in XDR $A$. baumannii pneumonia patients. This study may provide an insight view of XDR A. baumannii pneumonia clinical management during an era of stagnation in new antibiotic development.

Our study found that XDR A. baumannii pneumonia patients were more likely to have a smoking history and in elderly patients with complicated underlying disease and severer initial presentation ( higher APACHE II score). It was similar to previous studies[19-21]. Multivariate logistic regression analysis showed the APACHE II score greater than 20, COPD, and lower albumin levels were the independent risk factors for XDR A. baumannii pneumonia. Those findings indicated immunocompromised patients were more likely to develop XDR A. baumannii HAP. Using $\beta$-lactam/ $\beta$-lactamase inhibitors was also another independent risk factor. Clinicians need to be extra careful while using $\beta$-lactam/ $\beta$-lactamase inhibitors. XDR A. baumannii pneumonia patients may receive more invasive procedures due to severer clinical presentation. But medical devices may be the most contaminated locations for $A$. baumannii and caused hospital-acquired infections outbreaks [22,23]. Therefore, clinicians should be aware of invasive procedures uses and removing unnecessary accesses in time.

Previous studies had shown patients with antibiotic-resistant $A$. baumannii may have increased mortality compared with antibiotic-sensitive patients. [16, 24, 25]. The study in 2018 showed the increased mortality rate was associated with patient comorbidity and inappropriate therapy, but not antibiotic resistance[26]. And more studies revealed antibiotic resistance of $A$. baumannii was not associated with increase mortality. Sunenshine $\mathrm{RH}$, et al[16] reported mortality rate of MDR A. baumannii infection patients was not higher than non-MDR A. baumannii patients. Özgür et al[9] also found the mortality rate of patients with XDR A. baumannii VAP was not significantly higher than non-XDR A. baumannii patients in ICU settings. Aušra $C^{\vee}$ iginskiene, et al[12] reported the mortality rate in XDR A. baumannii VAP patients was not a significant difference compared with the non-XDR group. Zilberberg et al[17] examined the impact of carbapenem-resistance (CR) in patients with HAP and VAP in a large database of 8,969 patients. In this study, A. baumannii accounted for $11.8 \%$ of CR organisms and $2.5 \%$ of carbapenemsusceptible (CS) organisms and did not increase mortality for CR A. baumannii infection $(22.9 \%$ CR vs $21.6 \%$ CS). In our study, we found XDR A. baumannii pneumonia patients were associated with longer length of mechanical ventilation and hospital stay, but we did not observe increased in-hospital mortality. Those findings suggested resistance did not directly add to mortality and we may still improve patient outcomes if we optimize treatment with XDR A. baumannii pneumonia patients. 


\section{Conclusions}

XDR A. baumannii HAP was strongly related to systemic illnesses, invasive procedures, low albumin levels, the APACHE II score, longer length of mechanical ventilation, and hospital stay. But it did not increase in-hospital mortality.

\section{List Of Abbreviations}

A. baumannii, Acinetobacter baumannir; GFPH, Guangzhou First People's Hospital; FAH, the First Affiliated Hospital of Sun Yat-sen University; TAH, the Third Affiliated Hospital of Sun Yat-sen University; GRCH, Guangzhou Red Cross Hospital $\mathbb{G X}$ X, Guangzhou Xinhai Hospital; XDR, extremely drug-resistant; HAP, hospital-acquired pneumonia; VAP, ventilator-associated pneumonia; BAL, bronchoalveolar lavage; CRF, case record form; CRP, C-reactive protein; ESR, Erythrocyte sedimentation rate; PPIs, proton pump inhibitor drugs; CR, carbapenem-resistance; CS, carbapenem-susceptible.

\section{Declarations}

\section{Ethics approval and consent to participate}

The Guangzhou First People's Hospital Ethics Committee approved the study. Informed consents were not required as this was considered a review of clinical practice.

\section{Consent for publication}

Not applicable.

\section{Availability of data and materials}

The datasets used and/or analysed during the current study are available from the corresponding author on reasonable request.

\section{Competing interests}

The authors declare that they have no competing interests.

\section{Funding}

This work was funded by the Guangzhou Medical and Health Science and Technology Project (no. 20151A011004) and Guangzhou Science and Technology Project (No. 202002030036).

Natural Science Foundation of Guangdong Province (No. 2017A030313860). 
YjL supervised the study and wrote the manuscript. $\mathrm{XmH}$, WyY and $\mathrm{CzP}$ discussed the data and helped finalize the manuscript. ZxM, ZxZ, McH, CqF, HIC and PhG collected the data. ZwZ and SqW planned and supervised the experiments. All authors read and approved the final manuscript.

\section{Acknowledgements}

We thank Yongrou Yu, Guangdong Pharmaceutical University, Guangdong, China, for helping data acquisition.

\section{References}

1. Ozyurt S, Kostakoglu U, Yildiz IE, Erturk A, Sonmez E, Sahin U, Cicek AC: Investigation of the clonal associations in Acinetobacter Baumannii strains isolated from the respiratory samples of patients in a tertiary research hospital. Nigerian journal of clinical practice 2020, 23(8):1155-1162.

2. Boettger BC, Cayô R, Streling AP, Nodari CS, Almeida LGP, Martins W, Girardello R, Vasconcelos ATR, Gales AC, Pignatari ACC: Dynamic of High-Risk Acinetobacter baumannii Major Clones in a Brazilian Tertiary Hospital During a Short Time Period. Microbial drug resistance (Larchmont, NY) 2020.

3. Mohd Sazlly Lim S, Zainal Abidin A, Liew SM, Roberts JA, Sime FB: The global prevalence of multidrug-resistance among Acinetobacter baumannii causing hospital-acquired and ventilatorassociated pneumonia and its associated mortality: A systematic review and meta-analysis. The Journal of infection 2019, 79(6):593-600.

4. Abdallah M, Alsaleh H, Baradwan A, Alfawares R, Alobaid A, Rasheed A, Soliman I: Intraventricular Tigecycline as a Last Resort Therapy in a Patient with Difficult-to-Treat Healthcare-Associated Acinetobacter baumannii Ventriculitis: a Case Report. SN comprehensive clinical medicine 2020:1-5.

5. Nurtop E, Bayındır Bilman F, Menekse S, Kurt Azap O, Gönen M, Ergonul O, Can F: Promoters of Colistin Resistance in Acinetobacter baumannii Infections. Microbial drug resistance (Larchmont, NY) 2019, 25(7):997-1002.

6. Hu F, Guo $Y$, Yang $Y$, Zheng $Y$, Wu S, Jiang X, Zhu D: Resistance reported from China antimicrobial surveillance network (CHINET) in 2018. 2019, 38(12):2275-2281.

7. Garnacho-Montero J, Ortiz-Leyba C, Jiménez-Jiménez FJ, Barrero-Almodóvar AE, García-Garmendia JL, Bernabeu-Wittel IM, Gallego-Lara SL, Madrazo-Osuna J: Treatment of multidrug-resistant Acinetobacter baumannii ventilator-associated pneumonia (VAP) with intravenous colistin: a comparison with imipenem-susceptible VAP. Clinical infectious diseases : an official publication of the Infectious Diseases Society of America 2003, 36(9):1111-1118.

8. Batirel A, Balkan, II, Karabay O, Agalar C, Akalin S, Alici O, Alp E, Altay FA, Altin N, Arslan F et al: Comparison of colistin-carbapenem, colistin-sulbactam, and colistin plus other antibacterial agents for the treatment of extremely drug-resistant Acinetobacter baumannii bloodstream infections. European journal of clinical microbiology \& infectious diseases : official publication of the European Society of Clinical Microbiology 2014, 33(8):1311-1322. 
9. Özgür ES, Horasan ES, Karaca K, Ersöz G, Naycı Atış S, Kaya A: Ventilator-associated pneumonia due to extensive drug-resistant Acinetobacter baumannii: risk factors, clinical features, and outcomes. American journal of infection control 2014, 42(2):206-208.

10. Li YJ, Pan CZ, Fang CQ, Zhao ZX, Chen HL, Guo PH, Zhao ZW: Pneumonia caused by extensive drugresistant Acinetobacter baumannii among hospitalized patients: genetic relationships, risk factors and mortality. BMC infectious diseases 2017, 17(1):371.

11. Li Y, Pan C, Zhao Z, Zhao Z, Chen H, Lu W: Effects of a combination of amlodipine and imipenem on 42 clinical isolates of Acinetobacter baumannii obtained from a teaching hospital in Guangzhou, China. BMC infectious diseases 2013, 13:548.

12. Čiginskienè A, Dambrauskienè A, Rello J, Adukauskienè D: Ventilator-Associated Pneumonia due to Drug-Resistant Acinetobacter baumannii: Risk Factors and Mortality Relation with Resistance Profiles, and Independent Predictors of In-Hospital Mortality. Medicina (Kaunas, Lithuania) 2019, 55(2).

13. Gerson S, Lucaßen K, Wille J, Nodari CS, Stefanik D, Nowak J, Wille T, Betts JW, Roca I, Vila J et al: Diversity of amino acid substitutions in PmrCAB associated with colistin resistance in clinical isolates of Acinetobacter baumannii. International journal of antimicrobial agents 2020, 55(3):105862.

14. He T, Wang R, Liu D, Walsh TR, Zhang R, Lv Y, Ke Y, Ji Q: Emergence of plasmid-mediated high-level tigecycline resistance genes in animals and humans. 2019, 4(9):1450-1456.

15. Wang L, Liu D, Lv Y, Cui L, Li Y, Li T, Song H, Hao Y, Shen J, Wang Y et al: Novel Plasmid-Mediated tet(X5) Gene Conferring Resistance to Tigecycline, Eravacycline, and Omadacycline in a Clinical Acinetobacter baumannii Isolate. Antimicrobial agents and chemotherapy 2019, 64(1).

16. Sunenshine RH, Wright MO, Maragakis LL, Harris AD, Song X, Hebden J, Cosgrove SE, Anderson A, Carnell J, Jernigan DB et al: Multidrug-resistant Acinetobacter infection mortality rate and length of hospitalization. Emerging infectious diseases 2007, 13(1):97-103.

17. Zilberberg MD, Nathanson BH, Sulham K, Fan W, Shorr AF: A Novel Algorithm to Analyze Epidemiology and Outcomes of Carbapenem Resistance Among Patients With Hospital-Acquired and Ventilator-Associated Pneumonia: A Retrospective Cohort Study. Chest 2019, 155(6):1119-1130.

18. Horan TC, Andrus M, Dudeck MA: CDC/NHSN surveillance definition of health care-associated infection and criteria for specific types of infections in the acute care setting. American journal of infection control 2008, 36(5):309-332.

19. Dent LL, Marshall DR, Pratap S, Hulette RB: Multidrug resistant Acinetobacter baumannii: a descriptive study in a city hospital. BMC infectious diseases 2010, 10:196.

20. Zheng YL, Wan YF, Zhou LY, Ye ML, Liu S, Xu CQ, He YQ, Chen JH: Risk factors and mortality of patients with nosocomial carbapenem-resistant Acinetobacter baumannii pneumonia. American journal of infection control 2013, 41(7):e59-63.

21. Li Y, Guo Q, Wang P, Zhu D, Ye X, Wu S, Wang M: Clonal dissemination of extensively drug-resistant Acinetobacter baumannii producing an OXA-23 $\beta$-lactamase at a teaching hospital in Shanghai, 
China. Journal of microbiology, immunology, and infection = Wei mian yu gan ran za zhi 2015, 48(1):101-108.

22. Raro OHF, Gallo SW, Ferreira CAS, Oliveira SD: Carbapenem-resistant Acinetobacter baumannii contamination in an intensive care unit. Revista da Sociedade Brasileira de Medicina Tropical 2017, 50(2):167-172.

23. Garnacho-Montero J, Timsit JF: Managing Acinetobacter baumannii infections. Current opinion in infectious diseases 2019, 32(1):69-76.

24. Metan G, Sariguzel F, Sumerkan B: Factors influencing survival in patients with multi-drug-resistant Acinetobacter bacteraemia. European journal of internal medicine 2009, 20(5):540-544.

25. Al Jarousha AM, El Jadba AH, Al Afifi AS, El Qouqa IA: Nosocomial multidrug-resistant Acinetobacter baumannii in the neonatal intensive care unit in Gaza City, Palestine. International journal of infectious diseases : IJID : official publication of the International Society for Infectious Diseases 2009, 13(5):623-628.

26. Liang CA, Lin YC, Lu PL, Chen HC, Chang HL, Sheu CC: Antibiotic strategies and clinical outcomes in critically ill patients with pneumonia caused by carbapenem-resistant Acinetobacter baumannii. Clinical microbiology and infection : the official publication of the European Society of Clinical Microbiology and Infectious Diseases 2018, 24(8):908.e901-908.e907. 